\title{
Enhancement of Tumor Regression by Coulomb Nanoradiator Effect in Proton Treatment of Iron-Oxide Nanoparticle-Loaded Orthotopic Rat Glioma Model: Implication of Novel Particle Induced Radiation Therapy
}

\author{
Seung-Jun Seo ${ }^{1}$, Jae-Kun Jeon ${ }^{1}$, Eun-Ju Jeong ${ }^{2}$, Won-Seok Chang ${ }^{1,3}$, Gi-Hwan Choi ${ }^{4}$, Jong-Ki Kim ${ }^{{ }^{*}}$ \\ ${ }^{1}$ Department of Biomedical Engineering, School of Medicine, Catholic University of Daegu, 3056-6 Taemyung 4 Dong, Nam-Ku, \\ Daegu City, South Korea; ${ }^{2}$ Department of Diagnostic Imaging, School of Medicine, Catholic University of Daegu, 3056-6 Taemyung \\ 4 Dong, Nam-Ku, Daegu City, South Korea; ${ }^{3}$ Biomedical Engineering, College of Medicine, Kyungpook National University, 680 \\ Gukchaebosang-ro, Daegu, South Korea; ${ }^{4}$ Department of Neurosurgery, School of Medicine, Catholic University of Daegu, 3056-6 \\ Taemyung 4 Dong, Nam-Ku, Daegu City, South Korea. \\ Email: ${ }_{j}$ kkim@cu.ac.kr
}

Received November $12^{\text {th }}, 2013$; revised December $2^{\text {nd }}, 2013$; accepted December $9^{\text {th }}, 2013$

Copyright (C) 2013 Seung-Jun Seo et al. This is an open access article distributed under the Creative Commons Attribution License, which permits unrestricted use, distribution, and reproduction in any medium, provided the original work is properly cited.

\begin{abstract}
Background: Proton-impact metallic nanoparticles, inducing low-energy electrons emission and characteristic X-rays termed as Coulomb nanoradiator effect (CNR), are known to produce therapeutic enhancement in proton treatment on experimental tumors. The purpose of this pilot study was to investigate the effect of CNR-based dose enhancement on tumor growth inhibition in an iron-oxide nanoparticle (FeONP)-loaded orthotopic rat glioma model. Methods: Proton-induced CNR was exploited to treat glioma-bearing SD rat loaded with FeONP by either fully-absorbed single pristine Bragg peak (APBP) or spread-out Bragg peak (SOBP) 45-MeV proton beam. A selected number of rats were examined by MRI before and after treatment to obtain the size and position information for adjusting irradiation field. Tumor regression assay was performed by histological analysis of residual tumor in the sacrificed rats 7 days after treatment. The results of CNR-treated groups were compared with the proton alone control. Results: Intravenous injection of FeONP ( $300 \mathrm{mg} / \mathrm{kg})$ elevated the tumor concentration of iron up to $37 \mu \mathrm{g}$ of $\mathrm{Fe} / \mathrm{g}$ tissue, with a tumor-to-normal ratio of 5, 24 hours after injection. The group receiving FeONP and proton beam showed $65 \%-79 \%$ smaller tumor volume dose-dependently compared with the proton alone group. The rats receiving FeONP and controlled irradiation field by MR imaging demonstrated more than 95\% - 99\% tumor regression compared with MRI-determined initial tumor size. Conclusions: Proton-impact FeONP produced therapeutic enhancement compared with proton alone in an orthotopic rat glioma model at a selected temporal point after treatment. Single BP proton beam could induce CNRbased dose enhancement and produce enhanced tumor regression that was comparable to SOBP treatment despite inhomogeneous tumor dose in the APBP-treated tumor. These results may suggest emergence of novel Particle Induced Radiation Therapy (PIRT) on malignant glioma.
\end{abstract}

Keywords: Proton Therapy; Iron Oxide Nanoparticles; Coulomb Nanoradiator; Malignant Glioma

\section{Introduction}

Radiation therapy is a mainstay of treatment for patients with high grade gliomas, including glioblastoma. Radiation therapy in conjunction with surgery has been shown to prolong survival and, in the short term, improve cognitive function in patients with brain tumors. Over the

"Corresponding author. longer term, however, radiation can cause fatigue and serious, permanent side effects, including radiation necrosis. Proton beam therapy, on the other hand, delivers very precise, very high doses of radiation to a tumor site, while sparing the surrounding healthy tissue. Currently, most glioblastoma patients receive proton therapy with a dose standard of $60 \mathrm{~Gy}$, with concurrent chemotherapy. However, clinical trials suggest success and better overall 
survival rates with higher dosing [1,2]. The two-year overall survival rate for patients treated with the current standard of care is 26.5 percent. However, patients irradiated to 90 - $96.6 \mathrm{~Gy}$ (RBE) had a two-year overall survival rate of 34 - 45 percent.

Recently, our laboratory found proton-impact high- $Z$ nanoparticles produced CNR-based dose enhancement effect that led to a large therapeutic enhancement on nanoparticle-loaded mouse tumor model in either SOBP [3] or traversing Bragg peak irradiation [4]. Other groups also demonstrated the enhancement of cytotoxicity in their ion beam-impact in vitro studies on either platinum or gold-loaded cells $[5,6]$. Therapeutic enhancement was believed to have relevance to dose-enhancement effect from burst emission of low-energy electrons by Auger cascades of directly-impact ionized atom and interatomic relaxation process (IRP)-driven ionization from surrounding neutral atoms, collectively termed as Coulomb nanoradiator $(\mathrm{CNR})[4,7]$.

Here, we first report our pilot studies in nanoparticlesloaded orthotopic rat glioma model with the results of dose-dependent enhancement of tumor regression effect by proton-impact CNR. Importantly, a proton beam was applied in fully-absorbed single Bragg peak (APBP) to compare the effect of CNR-induction with conventional SOBP beam. The enhancement of tumor regression by APBP proton beam was comparable to the SOBP beam under the same dose. These observations may suggest emergence of novel particle induced radiation therapy (PIRT) on malignant glioma that may change present fractionation protocol in proton therapy or overcome the problem of tumor infiltration and radiation resistant population.

\section{Methods}

\subsection{Metal Nanoparticles}

Alginate-coated superparamagnetic magnetite nanoparticles (FeONP) were synthesized by insonating ferrous and ferric salt solutions, as reported previously $[8,9]$. Briefly, $\mathrm{FeCl}_{2} \cdot 4 \mathrm{H}_{2} \mathrm{O}(1.72 \mathrm{~g})$ and $\mathrm{FeCl}_{3} \cdot 6 \mathrm{H}_{2} \mathrm{O}(4.70 \mathrm{~g})(8.65$ mmol Fe${ }^{2+} / 17.30 \mathrm{mmol} \mathrm{Fe}{ }^{3+}$ ) were dissolved in $80 \mathrm{ml}$ of distilled water. A black magnetic oxide precipitate was obtained by heating the solution to $80^{\circ} \mathrm{C}$ in argon atmosphere, increasing the $\mathrm{pH}$ to 10 by adding $28 \%-30 \%$ ammonium hydroxide to the water, and insonating the mixed iron solution with $20-\mathrm{kHz}$ ultrasound at a power output of $140 \mathrm{~W}$ for $1 \mathrm{~h}$. Alginate was used to coat the nanoparticle surface to disperse the particles. Briefly, $2 \mathrm{~g}$ of magnetite nanoparticles were dispersed in $60 \mathrm{ml}$ of saline and $25 \mathrm{ml}$ of alginic acid solution by heating the solution to $80^{\circ} \mathrm{C}$ while insonating at power output of $50 \mathrm{~W}$ for
30 min under nitrogen gas with continuous stirring. The particles were purified by washing with saline while being exposed to a strong neodymiummagnet (magnetic field density; $B_{r}=11,000$ Gauss). Finally, a ferrofluid containing $25 \mathrm{mg} / \mathrm{ml} \mathrm{FeONP}$ was obtained.

\subsection{Transmission Electron Microscopy (TEM) Studies}

The average particle size, size distribution, and morphology of FeONP were examined using a Zeiss 902 transmission electron microscope (Carl Zeiss Pte., Ltd., Oberkochen, Germany) at a voltage of $80 \mathrm{kV}$. The aqueous dispersion of the particles was drop casted onto a carboncoated copper grid, and the grid was air dried at room temperature before microscopic observation.

\subsection{Animal Models}

Intracranial gliomas were prepared by inoculating $5 \times$ $10^{6} \mathrm{C} 6$ glioma cells stereotactically $5 \mathrm{~mm}$ deep into the frontal lobe of the left hemisphere of Sprague Darley (SD) rats after a craniotomy, as described elsewhere [10]. The animals were anesthetized by intraperitoneal injections of ketamine and xylazine at $60 \mathrm{mg} \cdot \mathrm{kg}^{-1}$. After immobilizing the rats in a rodent stereotactic frame, an incision was made in the skin and a burr hole made in the skull. One million tumor cells were injected at a rate of 1 - 2 microliters/minute using a microsyringe (Hamilton, Reno, NV, US) mounted on a stereotactic frame (Kopf Instruments, Tujunga, CA, US), at coordinates of $1 \mathrm{~mm}$ lateral and 1 $\mathrm{mm}$ posterior to the bregma and $1.5 \mathrm{~mm}$ below the dura. The incision was closed with veterinary adhesive and topical lidocaine was administered.

\subsection{Tumoral Uptake of Nanoparticles}

To measure nanoparticle uptake in the tumors, nanoparticle doses of either 100 or $300 \mathrm{mg} / \mathrm{kg}$ body weight were administered to the $\mathrm{C} 6$ tumor models via the tail vein $1 \mathrm{~h}$ or $24 \mathrm{~h}$ prior to surgical removal of the tumors. In addition, normal brain tissue was also sampled to measure tumor-to-normal ratio. Tumor and normal samples were placed in tared vials and analyzed for iron using ICP-MS spectrometry.

\subsection{MRI Examination}

MRI imaging at a $1.5 \mathrm{~T}$ MRI unit (GE $1.5 \mathrm{~T}$, US) was performed on selected rats to confirm the formation of gliomas seven days after the implantation of the C6 cells. Brain axial T1-weighted images were acquired using a wrist coil and a fast spin echo sequence (FSE) to monitor the formation of the tumor mass after injecting a contrast agent at a dose of $1 \mathrm{mg} / \mathrm{kg}$. Scan parameters of the FSE 
Treatment of Iron-Oxide Nanoparticle-Loaded Orthotopic Rat Glioma Model: Implication of Novel Particle Induced Radiation Therapy

imaging were set as follows: an echo time of $15 \mathrm{~ms}$, a repetition time of $450 \mathrm{~ms}$, a field of view (FOV) of 80 $\mathrm{mm}$, an imaging matrix of $320 \times 256$, a slice thickness of $2 \mathrm{~mm}$, an ETL of 4, and a number of excitations (NEX) of 4 .

\subsection{Proton Irradiation Experiments}

The $45 \mathrm{MeV}$ proton beam irradiations were conducted in the animal model using the experimental setup presented in Figure 1 at the Korea Cancer Center Hospital (Seoul, Korea) while delivering an average proton dose with a dose rate of $0.51-0.67 \mathrm{~Gy} / \mathrm{s}$ to the sample. Fifty microliters of iron NPs in saline solution were administered in the tail vein of the animals $24 \mathrm{~h}$ prior to proton irradiation. The proton beam irradiated the orthotopic rat glioma model in either SOBP or a fully-absorbed manner with a single BP (APBP) occurrence at the greatest depth of the tumor volume as shown in Figure 1 with two-single doses, 20 or $40 \mathrm{~Gy}$. In this single BP irradiation, the proximal tumor volume was exposed to the plateau dose (PD). Thus, the tumor mass was treated by CNR plus either BP or PD.

Three experimental groups of C6 rat glioma models were prepared for Treatment-APBP and Treatment-SOBP, respectively, by intravenously injecting FeONPs at 300

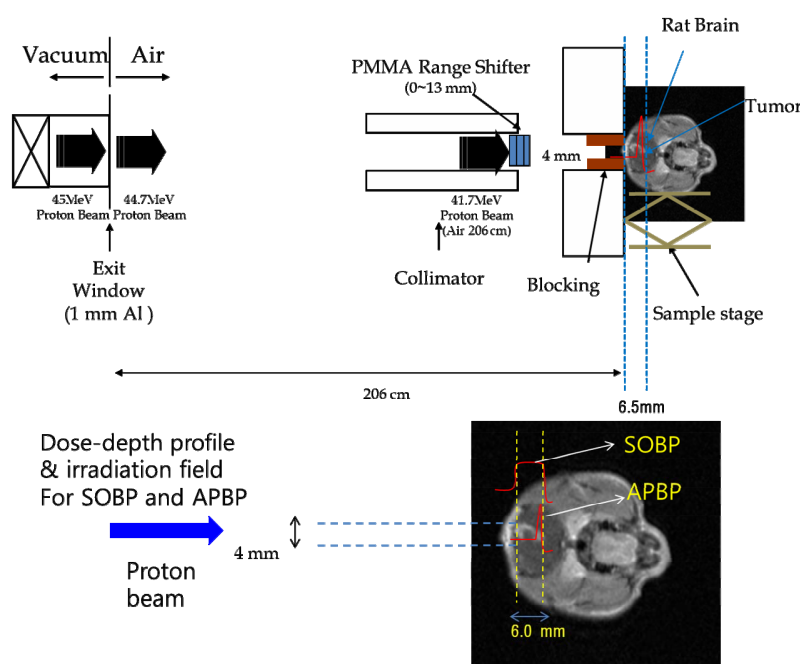

Figure 1. The experimental layout describes the appropriate irradiation mode with different Bragg peak positions. The proton beam energy after the $\mathrm{Al}$ window was $40 \mathrm{Me}-$ Vinthe collimator. For Treatment-APBP, a single pristine Bragg peak was placed at $6 \mathbf{m m}$ depth of the tumor in the rat brain. For Treatment-SOBP, 40 Gy was delivered homogeneously up to the estimated average tumor depth of $6 \mathbf{m m}$ andthe tumor area of $4 \mathrm{~mm}$ based on a separate histological analysis of initial tumor size; the surrounding normal tissues were shielded using a series of acrylate blocks and a bolus positioned inside the beam collimator to fit the tumor size. $\mathrm{mg} / \mathrm{kg}$ body weight into the rat. Five proton-alone groups were also prepared for each proton dose as controls. The rats were anesthetized by the intraperitoneal injection of $20 \mathrm{mg} / \mathrm{kg}$ ketamine and $18.4 \mathrm{mg} / \mathrm{kg}$ xylazine. For Treatment-APBP, each animal group was irradiated with a single dose proton beam in which either 20 or 40 Gy was delivered at BP from tumor depth of $6 \mathrm{~mm}$. In Treatment-SOBP, 40 Gy was delivered homogeneously up to the estimated typical tumor depth of $6 \mathrm{~mm}$ and the tumor area of $4 \mathrm{~mm}$ that was determined by histological measurement of average initial tumor size 7 days after implanting tumor cell in separate five rats; the surrounding normal tissues were shielded using a series of acrylate blocks and a bolus positioned inside the beam collimator to fit the tumor size. Therefore, homogeneity of proton dose in Treatment-SOBP was assured only in the central region of tumor mass, $4 \times 6 \mathrm{~mm}$. Radiation dose might not be delivered in some parts where initial tumor mass was larger than this estimated typical irradiation field, because proton beam was not irradiated in stereotactic manner with guidance by MRI -based tumor position in each animal. Only selected numbers of rat were examined using MRI before proton irradiation to fit irradiation field according to obtained size and depth. In these cases, initial tumor size before treatment was estimated from MRI data.

\subsection{Tumor Regression Assay and Statistical Analysis}

Each animal group was killed 7 days after the treatment by an overdose injection of sodium pentobarbital. The brains were removed, fixed in $10 \%$ formaldehyde, paraffin embedded, and sectioned through the area of irradiation. The 5 - $\mu \mathrm{m}$-thick sections were stained with hematoxylin and eosin, and the tumor was examined microscopically. For each rat, the largest lesion area was measured by a microscope with image analysis software (Axitophot, Zeiss, Germany). The tumor shape was assumed to approximate a spheroid. The volume was calculated using the formula $4 \pi / 3 \times x / 2 \times y / 2 \times z / 2: x$ and $y$ are the dimensions of the largest lesion area while $\mathrm{z}$ is the height of each section $(5-\mu \mathrm{m})$ multiplied by the number of sections containing the tumor tissue. In case residual tumor was minimal and irregular, the area of each tumor section was calculated using image analysis software to integrate for total tumor volume. The therapeutic response was evaluated by histological assay, measuring the tumor size of each experimental group after the animals were sacrificed and compared with the average tumor size before treatment and the proton alone group. The differences between the groups were assessed with a one-way ANOVA followed by the Bonferroni multiple compari- 
son test. A p value of $<0.05$ was considered the level of significance for all of our tests. All data were analyzed using a contemporary statistical software package (GraphPad Prism ${ }^{\mathrm{TM}}$; GraphPad Software, Inc., San Diego, CA, USA).

\section{Results}

\subsection{Size Distribution Studies by TEM Measurements}

The average size of the particles was determined by TEM, by using measurements of the size of approximately 200 particles. The particles had a globular shape and an approximate size of $10.6 \mathrm{~nm}$ with a standard deviation of $0.78 \mathrm{~nm}$. The size of the particles after coating was 13 $15 \mathrm{~nm}$ in diameter.

\subsection{Tumor Uptake}

ICP-MS data are summarized in Table 1. Tumor concentrations of FeONP $24 \mathrm{~h}$ after injection with a dose of 300 $\mathrm{mg} / \mathrm{kg}$ were $37.6 \pm 6.3 \mu \mathrm{g} \cdot \mathrm{Fe} / \mathrm{g}$ tissue, while the corresponding normal were $7.4 \pm 3.7 \mu \mathrm{g} \cdot \mathrm{Fe} / \mathrm{g}$ tissue. The tumor-to-normal $\mathrm{FeONP}$ ratio was about 5 after 24-hour post-injection. When iron nanoparticles were injected, less than $1 \%$ of the injected dose was taken by tumor in a given time interval after injection.

\subsection{Tumor Regression Effect of Proton-Impact Nanoparticles on FeONP Rats}

The mean tumor sizes of various groups were summarized for each treatment group in Table 2. The average tumor size was significantly different in the rat receiving only proton radiation and those receiving $\mathrm{FeONP}$ followed by either SOBP $(p<0.05)$ or APBP $(p<0.02)$ proton irradiation, as shown in Table 2. The FeONPSOBP rat demonstrated smallest average tumor size among experimental groups 7 days after treatment and significantly $(p<0.05)$ smaller tumor size compared to the SOBP-proton alone rat. The FeONP-APBP rat also showed comparable (but statistically insignificant) tumor volume regression to the FeONP-SOBP rat after irradiation with $40 \mathrm{~Gy}$, and the average tumor size decreased dosedependently between 20 - $40 \mathrm{~Gy}$.

Although only small numbers of rats were examined by MRI before treatment to measure the tumor size and position, enabling conformal energy delivery by fitting the irradiation field to the tumor volume information, the FeONP-MRI rats showed much better tumor regression compared with corresponding un-examined groups shown in Table 2. T1-weighted MRI-data of some se-

Table 1. Results of tumor uptake following the injection of iron-oxide nanoparticles injection dose.

\begin{tabular}{|c|c|c|c|c|}
\hline & Tissue & & & $100 \mathrm{mg} / \mathrm{kg}$ \\
\hline \multirow{3}{*}{ Tissue concentration ( $\mu \mathrm{g}$ of $\mathrm{Fe} / \mathrm{g}$ tissue) } & & $1 \mathrm{~h}$ & $24 \mathrm{~h}$ & $24 \mathrm{~h}$ \\
\hline & Tumor & $26.2 \pm 4.1$ & $37.6 \pm 6.3$ & $19.6 \pm 5.4$ \\
\hline & Normal & $12.5 \pm 3.8$ & $7.4 \pm 3.7$ & \\
\hline Uptake/injection (\%) & & & 0.6 & \\
\hline
\end{tabular}

Table 2. The mean tumor sizes were summarized for each treatment group.

\begin{tabular}{|c|c|c|c|c|c|}
\hline \multirow[t]{2}{*}{ Mean TV } & \multicolumn{4}{|c|}{ Proton dose (at BP) } & \multirow[b]{2}{*}{$\mathrm{P}^{*}$} \\
\hline & 0 & 40 Gy (SOBP) & 40 Gy (APBP) & 20 GY (APBP) & \\
\hline \multicolumn{6}{|l|}{ FeONP (mg/kg) } \\
\hline Baseline & $50.2 \pm 25.2(\mathrm{n}=5)$ & & & & \\
\hline 0 (proton alone) & & $21.3 \pm 7.2(\mathrm{n}=5)$ & $38.1 \pm 20.2(\mathrm{n}=6)$ & $52.7 \pm 21.7(\mathrm{n}=6)$ & 0.007 \\
\hline 300 & & $3.1 \pm 2.6(\mathrm{n}=5)$ & $8.6 \pm 6.6(n=5)$ & $18.9 \pm 10.2(\mathrm{n}=5)$ & \\
\hline (MRI-ex) Baseline & & 76.8 & $49.2 \pm 16.6$ & $58.5 \pm 8.4$ & 0.014 \\
\hline (MRI-ex) 300 & & $1.5(\mathrm{n}=1)$ & $0.8 \pm 0.9(\mathrm{n}=2)$ & $9.3 \pm 5.6(\mathrm{n}=2)$ & \\
\hline $\mathrm{p}$ & & 0.036 & 0.013 & 0.021 & \\
\hline
\end{tabular}

The $p$ value refers to the significance of the difference among two or three rat groups treated with Treatment-APBP or Treatment-SOBP across columns or rows ${ }^{*}$, respectively using ANOVA analysis. MRI-rats were not included in calculating $p$ value across column. 
lected FeONP-rats using contrast agent showed the hyper-intense tumor area in pre-treatment MRI that were minimal or reduced clearly in post-treatment MRI of the FeONP rats by three different treatments; APBP-20 Gy, APBP-40 Gy, SOBP-40 Gy as shown in Figure 2. Each histological image, obtained from sacrificed rats after treatment, demonstrated the correspondence with its MRI data.

\section{Discussion}

Despite potent unmatched tumor dose distribution to actual tumor size in this non-stereotactic proton irradiation studies on orthotopic glioma model, it was attempted to elucidate the effect of proton-impact nanoparticles within tumor mass on tumor regression by comparing the combined nanoparticle and proton experiment with the proton alone experiment at a given days after treatment. Statistically-significant enhanced growth inhibition effect from the FeONP rats indicates that this protocol was able to produce CNR effect, enabling the rapeutic enhancement at clinically-relevant proton dose despite insufficient precision in delivering tumor dose. However, relatively smaller fluctuation in average tumor size was observed in CNR-producing experimental group compared with the proton alone groups. MRI-examined FeONP rats demonstrated more than $90 \%$ tumor regression 7 days after treatment that may be attributed to relatively well-matched tumor dose to the tumor size and position. Other FeONP rats showed more than $60 \%$ smaller average residual tumor size compared with the proton alone, led to more than $62 \%$ tumor regression with respect to the average initial tumor size. Conversely, growth retardation or $24 \%-50 \%$ regression effect was observed in the proton alone group. Dose-dependently decreased tumor size after Treatment-APBP on the FeONP rats suggested that CNR-mediated therapeutic effects increased also dosedependently as observed in previous studies with mice Xenograft tumor model [4]. Comparable tumor regression effect of the APBP-FeONP rats to the SOBP proton alone suggested that single BP proton beam combined with CNR could deliver effectively similar tumor dose with SOBP proton beam. This effect may be exploited to modify current fractionation protocol in proton treatment of brain tumor without enhanced entrance dose. Such enhancement under the inhomogeneous distribution of tumor dose may be attributed to both low-energy CNR electrons $(0-1000 \mathrm{eV})$ and concomitant enhancement of ROS generation with energy-dependent migration mobility of related ROS molecules [4]. Low energy electrons can transport up to several hundred nm, while converted ROS diffuse up to about several $\mu$ [11].

The CNR effects under a low dose primary irradiation suggests not only dose enhancement within the tumor but also less side effects to the surrounding normal compared to therapeutic high-dose irradiation alone, effectively tumor-specific. This enhanced tumor dose with less normal dose may provide an novel Particle Induced Radiation Therapy (PIRT) with important therapeutic outcome such as longer survival and less side effects in proton treatment of brain tumor where radiation treatment interferes with many neuronal function and pediatric brain development. In addition, PIRT may modulate greatly present fractionation scheme, leading to shortening total treatment period. The efficiency of radiation therapy is often hindered by diffusively invasive characteristic of brain tumor as well as emergence of radiation-resistant population. On the other hand, PIRT with traversing Bragg peak can be exploited potentially to treat the tumor spreading in normal as long as iron nanoparticles are preferentially taken in tumor cell, which are under way in our laboratory. Present tumor-to-normal ratio of iron-oxide NP in this study could be obtained from either EPR effect or facilitated BBB crossing by macrophage-uptake of NP $[12,13]$. Although concentration of nanoparticles with about $40 \mu \mathrm{g} \cdot \mathrm{Fe} / \mathrm{g}$ tissue was achieved injection-dose dependently in this study, less than $1 \%$ of total dose was taken up in brain tumor. Thus intravenous injection of $300 \mathrm{mg} / \mathrm{kg}$ would be too much for clinical practice in human. In this regard, targeted NP with BBB-crossing and radiation-enhanced $\mathrm{BBB}$ disruption [14] may further increase tumoral uptake of NP even in the tumor infiltration of malignant glioma where it often occurs at intactBBB normal tissue. This advance in nanotechnology combined with energy-delivery by high-energy ion beam may enable tumor control of malignant glioma without side effects.

Nanoradiator effect can be obtained from either Coulomb collision with ion beam or photoelectric absorption of X-ray photon. However, ion-beam irradiation may provide much better way in the energy delivery as well as Z-value independent interaction with nanoparticles compared with X-ray photon under the normal tissue tolerance $[5,15]$.

\section{Conclusion}

In conclusion, proton-impact FeONP produced therapeutic enhancement in an orthotopic rat glioma model by either conventional SOBP or single pristine Bragg peak irradiation. The results suggested emergence of novel Particle Induced Radiation Therapy (PIRT) on malignant glioma that may enable treatment of tumor infiltration or shortening current fractionation period without radiation resistant population. 
Implication of Novel Particle Induced Radiation Therapy
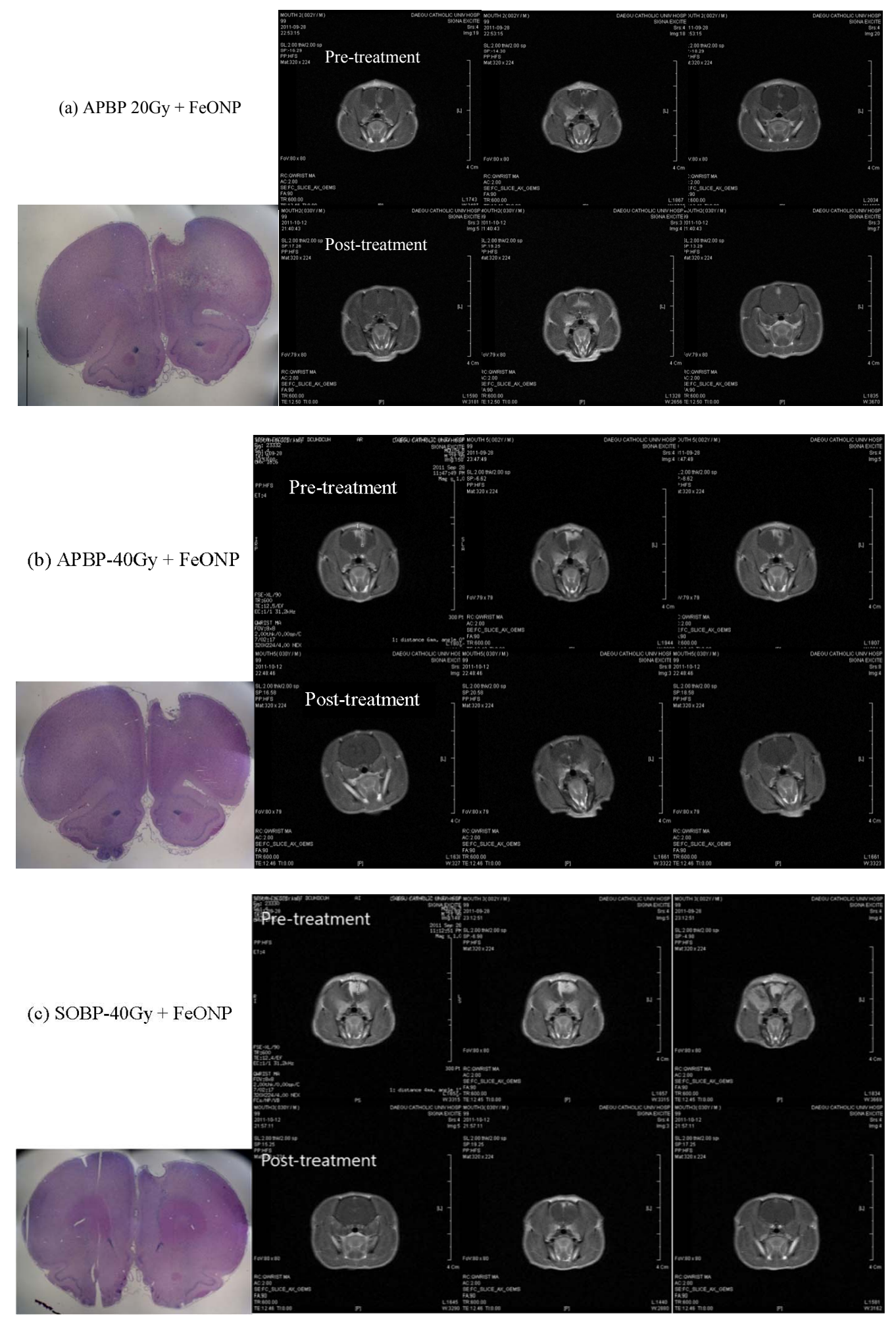

Figure 2. MRI-data of some selected FeONP-rats were acquired one day before and 7 days after proton treatment using contrast agent-based T1-weighted imaging, leading to hyper-intense tumor area. This enhanced tumor region in pre-treatment MRI were minimal or reduced clearly in post-treatment MRI of three different treatments; APBP-20 Gy (a), APBP-40 Gy (b), SOBP-40 Gy on the FeONP rat. Each histological image was obtained from sacrificed rats after treatment and MRI examination. 


\section{Acknowledgments}

This research was supported by Atomic foundation expansion program, the National Research Foundation of Korea (NRF-2012M2B2A4029568) funded by the Ministry of Education, Science and Technology, and partially by the Catholic University of Daegu (20135001).

\section{REFERENCES}

[1] M. M. Fitzek, A. F. Thornton, J. D. Rabinov, et al., “Accelerated Fractionated Proton/Photon Irradiation to $90 \mathrm{Co}-$ balt Gray Equivalent for Glioblastoma Multiforme: Results of a Phase II Prospective Trial," Journal of Neurosurgery, Vol. 1, No. 2, 1999, pp. 251-260. http://dx.doi.org/10.3171/jns.1999.91.2.0251

[2] M. Mizumoto, K. Tsuboi, H. Igaki, et al., "Phase I/II Trial of Hyperfractionated Concomitant Boost Proton Radiotherapy for Supratentorial Glioblastoma Multiforme" International Journal of Radiation Oncology Biology Physics, Vol. 77, No. 1, 2010, pp. 98-105. http://dx.doi.org/10.1016/j.ijrobp.2009.04.054

[3] J.-K. Kim, S.-J. Seo, H.-T. Kim, K.-H. Kim, M.-H. Chung and K.-R. Kim, "Therapeutic Application of Metallic Nanoparticles Combined with Particle-Induced XRay Emission Effect," Nanotechnology, Vol. 21, No. 42, 2010, Article ID: 425102. http://dx.doi.org/10.1088/0957-4484/21/42/425102

[4] J.-K. Kim, S.-J. Seo, H.-T. Kim, K.-H. Kim, M.-H. Chung and K.-R. Kim, "Enhanced Proton Treatment in Mouse Tumors through Proton Irradiated Nanoradiator Effects on Metallic Nanoparticles," Physics in Medicine and Biology, Vol. 57, No. 24, 2012, pp. 8309-8323. http://dx.doi.org/10.1088/0031-9155/57/24/8309

[5] P. C. Polf, L. F. Bronk, W. H. F. Driessen, W. Arap, R. Pasqualini and M. Gillin, "Enhanced Relative Biological Effectiveness of Proton Radiotherapy in Tumor Cells with Internalized Gold Nanoparticles," Applied Physical Letters, Vol. 98, No. 19, 2011, Article ID: 193702. http://dx.doi.org/10.1063/1.3589914

[6] E. Porcel, S. Liehn, H. Remita, N. Usami, K. Kobayashi and Y. Furusawa, "Platinum Nanoparticles: A Promising Material for Future Cancer Therapy?" Nanotechnology, Vol. 21, No. 8, 2010, Article ID: 085103. http://dx.doi.org/10.1088/0957-4484/21/8/085103

[7] J.-K. Kim, S.-J. Seo, T.-J. Kim, K. Hyodo, A. Zaboronok, H. You, K. Peach and M. A. Hill, "Enhanced Generation of Reactive Oxygen Species by the Nanoradiator Effect from Core-Inner Shell Photo-Excitation or Proton Impact on Nanoparticle Atomic Clusters," Radiation Research, 2013 submitted.

[8] S.-I. Park, J.-H. Lim, Y.-H. Hwang, J.-H. Kim, C.-G. Kim and C.-O. Kim, "In Vivo and in Vitro Antitumor Activity of Doxorubicin-Loaded Magnetic Fluids," Physica Status Solidi (c), Vol. 4, No. 12, 2007, pp. 4345-4451.

[9] R. Vijayakumar, Y. Koltypin, I. Felner and A. Gedanken, "Sonochemical Synthesis and Characterization of Pure Nanometer-Sized $\mathrm{Fe}_{3} \mathrm{O}_{4}$ Particles," Materials Science and Engineering: A, Vol. 286, 2000, pp. 101-105. http://dx.doi.org/10.1016/S0921-5093(00)00647-X

[10] S.-J. Seo, N. Sunaguchi, T. Yuasa, Q. Huo, M. Ando, G.H. Choi, H.-T. Kim, W.-S. Chang, K.-H. Kim, E.-J. Jeong and J.-K. Kim, "Visualization of Microvessel Proliferation as a Tumor Infiltration Structure in Rat Glioma Specimens Using the Diffraction-Enhanced Imaging In-Plane CT Technique," Physics in Medicine and Biology, Vol. 57, No. 5, 2012, pp. 1251-1262. http://dx.doi.org/10.1088/0031-9155/57/5/1251

[11] J. D. Carter, N. N. Cheng, Y. Qu, G. D. Suarez and T. Guo, "Nanoscale Energy Deposition by X-Ray Absorbing Nanostructures," Journal of Physical Chemistry B, Vol. 111, 2007, pp. 11622-11625. http://dx.doi.org/10.1021/jp075253u

[12] P. Ballabh, A. Praun and M. Nedergaard, "The Blood Brain Barrier: An Overview Structure, Regulation, and Clinical Implications," Neurobiology of Diseases, Vol. 16, No. 1, 2004, pp. 1-13. http://dx.doi.org/10.1016/j.nbd.2003.12.016

[13] H. Wolburg, K. Wolburg-Buchholz and B. Engelhardt, "Diapedesis of Mononuclear Cells across Cerebral Venules during Experimental Autoimmune Encephalomyelitis Leaves Tight Junctions Intact," Acta Neuropathology, Vol. 109, No. 2, 2005, pp. 181-190. http://dx.doi.org/10.1007/s00401-004-0928-x

[14] D. Y. Joh, L. Sun, M. Stangl, A. Al Zaki, S. Murty, P. P. Santoiemma, J. J. Davis, B. C. Baumann, M. Alonso-Basanta, D. Bhang, G. D. Kao, A. Tsourkas and J. F. Dorsey, "Selective Targeting of Brain Tumors with Gold Nanoparticle-Induced Radiosensitization," PLoS One, Vol. 30, No. 8, 2013, Article ID: e62425. http://dx.doi.org/10.1371/journal.pone.0062425

[15] G.-H. Choi, S.-J. Seo, K.-H. Kim, H.-T. Kim, S.-H. Park, J.-H. Lim and J.-K. Kim, "Photon Activated Therapy (PAT) Using Monochromatic Synchrotron X-Rays and Iron Oxide Nanoparticles in a Mouse Tumor Model: Feasibility Study of PAT for the Treatment of Superficial Malignancy," Radiation Oncology, Vol. 7, 2012, p. 184. http://dx.doi.org/10.1186/1748-717X-7-184 


\section{Abbreviations}

NP: Nanoparticle;

CTR: Complete Tumor Regression;

ROS: Reactive Oxygen Species;

CNR: Coulomb Nanoradiator Effect;

PIR: Particle Induced Radiation;

PIRT: PIR Therapy;
SOBP: Spread-Out Bragg Peak;

BP: Bragg Peak;

APBP: Fully Absorbed Pristine Bragg Peak;

Treatment-APBP: PIRT with Fully Absorbed Single

Bragg Peak;

Treatment-SOBP: PIRT with Spread-Out Bragg Peak; IRP: Interatomic Relaxation Process. 